\section{As necessidades comunicacionais das práticas educativas na prevenção da transmissão materno-fetal do HIV}

\section{Communication needs of education practices in preventing maternal-fetal HIV transmission}

Katia Virginia de O Feliciano 1

Maria Helena Kovacs 2

${ }^{1}$ Secretaria de Saúde de Pernambuco. Praça Oswaldo Cruz, s. n. Recife, PE, Brasil. CEP: 50.000-110

2 Faculdade de Ciências Médicas, Universidade de Pernambuco

\begin{abstract}
Objectives: to determine the efficiency of educational methods in preventing maternal-fetal AIDS transmission through communication among users and health services providers.

Methods: exploratory study, part of the survey on pre-natal care, accomplished in 19 clinics, two maternity hospitals and six family health units performed by the Health Secretariat of the city of Recife, from May to July, 1998. Procedures were observed, technical teams were interviewed and questionnaires applied to 355 women being seen, of these, 81 participated of the survey.

Results: healthcare providers do not perform counseling, even when informing on HIV testing $(48,1 \%)$. Education work is focused on furnishing information during consultation, for, collective activities in the units doing them $(48,1 \%)$ do not occur in all of the pre-natal care schedules. Education based on strategic rationality underestimates the value of women's informed choice of which only 11,1\% used condoms o a systematic way.

Conclusions: insufficient education during prenatal care and a "technocratic monologue" prevailing on the relationship between users and healthcare providers have a negative impact on the vulnerability of the female population and their children.
\end{abstract}

Key words Acquired Immunodeficiency Syndrome, Prenatal care, Communication, Health education

\section{Resumo}

Objetivos: elucidar a fecundidade da prática educativa centrada na prevenção da transmissão maternofetal da AIDS para promover a comunicação usuárias-serviços.

Métodos: estudo exploratório, parte de uma pesquisa sobre atenção pré-natal, realizado em 19 centros de saúde, duas maternidades e seis unidades de saúde da família da Secretaria de Saúde da cidade do Recife, de maio a julho de 1998. O processo de trabalho foi observado, equipes técnicas entrevistadas e um questionário foi aplicado às 355 mulheres em atendimento; dessas, 81 foram submetidas à sondagem.

Resultados: nenhum serviço faz aconselhamento, mesmo quando informam sobre o teste anti-HIV (48,1\%). O trabalho educativo está focalizado no fornecimento de informações durante a consulta, pois, as atividades coletivas, nas unidades que realizam-nas (48,1\%), não ocorrem em todos os horários do prénatal. A prática educativa baseada na racionalidade estratégica subestima a importância da aproximação às decisões valorativas das mulheres, das quais somente 11,1\% utilizam camisinha de modo sistemático.

Conclusões: a pequena oferta de atividades educativas no pré-natal e o "monólogo tecnocrático" que predomina nas relações entre usuárias e serviços de saúde têm repercussões negativas na vulnerabilidade da população feminina e de seus filhos.

Palavras-chave Síndrome de Imunodeficiência Adquirida, Cuidado pré-natal, Comunicação, Educação em saúde 


\section{Introdução}

A mudança do perfil epidemiológico da Human Immunodeficiency Vírus (HIV), Síndrome de Imunodeficiência Adquirida (AIDS), traduzida pelo aumento do número de casos no segmento feminino da população inicialmente identificado como não exposto - mulheres que têm um único parceiro e não usam drogas - e no conseqüente crescimento da transmissão vertical do vírus, propiciou um maior interesse pelas mulheres grávidas. Desde 1995, o Ministério da Saúde prioriza a gestação como o momento para diagnosticar a infecção pelo vírus (submeter-se ou não ao teste anti-HIV, torna-se uma decisão que os serviços devem solicitar às mulheres que procuram atenção pré-natal) tratar as soropositivas com drogas anti-retrovirais e fornecer orientações sobre a alimentação dos bebês e a prática de sexo seguro. ${ }^{1}$ A resposta institucional, ao minimizar o fato de que a AIDS diz respeito a todas as mulheres e reforçar uma organização do trabalho que dissocia as várias dimensões da saúde reprodutiva feminina, acentua a preocupação com as características das práticas educativas desenvolvidas no cotidiano dos serviços de saúde.

A educação em saúde voltada para a prevenção da AIDS, nos últimos anos, tem sido submetida a questionamentos acerca dos seus pressupostos teóricometodológicos dominantes, sobressaindo-se as críticas sobre as insuficiências da abordagem focalizada na tentativa de conscientizar as pessoas sobre a própria suscetibilidade, responsabilizando-as pela transformação dos comportamentos que aumentam a probabilidade de infecção pelo vírus.2,3 Deste modo, são escamoteadas as relações sociais, econômicas, culturais e afetivas que configuram os distintos contextos de intersubjetividade, nos quais as pessoas desenvolvem formas diferenciadas de interpretação e valorização das necessidades de saúde, assim como, de apropriação dos conhecimentos sobre as alternativas para satisfazê-las. Esta desvalorização dos significados atribuídos à vivência da sexualidade e à proteção contra a AIDS é reiterada pelo caráter unidirecional das práticas educativas, convertidas em atos de transferência de informações.

Até hoje, de acordo com o ideário preconizado pelo Departamento Nacional de Saúde Pública a educação em saúde, desde a década de 20, as concepções hegemônicas nas práticas de comunicação em saúde têm recorrido ao uso dos meios como possibilidade de persuasão, buscando responder a um certo "espírito de relutância" das pessoas e dos grupos sociais em aderir às políticas, programas e conhecimentos previamente definidos. Com o intuito de conferir uma maior eficácia à transmissão de informações, a partir dos anos 70, tem sido feita a tentativa de estabelecer um "código comum" por meio do "ajuste da linguagem" utilizada pelos profissionais no trabalho educativo à linguagem cotidiana da "população-alvo", tornando-a, assim, mais adequada para a difusão do conteúdo das mensagens institucionais. 4,5

Esse modelo de comunicação, que tem a pretensão de exercer influência sobre a definição da situação ou sobre a decisão do outro, explicita o predomínio da racionalidade estratégica na esfera das ações de saúde. Embora essas ações articulem duas dimensões que são praticadas em conjunto, a técnico-científica e a relacional, 6 o fato dessa relação ocorrer entre pessoas que no plano social apresentam autoridades técnicas e científicas muito desiguais, tem permitido caracterizá-la como uma ação solitária de um sujeito que quer conhecer e agir sobre objetos. Referida a meios e fins da ação social, essa racionalidade de caráter fundamentalmente tecnológico, do saber fazer, que determina a priori as formas possíveis de recriação do mundo, não apenas reprime a satisfação das necessidades, mas tende a reprimir a própria emergência de necessidades. ${ }^{7}$

Foi refletindo sobre as conseqüências da progressiva influência do desenvolvimento científico e tecnológico na vida prática que Habermas, ${ }^{7}$ mesmo reconhecendo a importância da ciência e da técnica para a vida humana, desenvolveu a idéia de "colonização do mundo da vida". Ou seja, do avanço da racionalidade técnica sobre o celeiro cultural que armazena as competências gerais, os modelos de interpretação e o saber de regras, necessários aos processos comunicativos voltados ao entendimento. Essa atitude cria obstáculos para a atividade crítica dirigida para o conhecimento e a ação, portanto, para o processo de avaliar e emitir juízos sobre as relações entre o que vale como conhecimento (discurso teórico) e a interpretação das necessidades à luz das informações existentes e das experiências vividas (discurso prático), etapa essencial para o estabelecimento de um acordo por meio do qual as pessoas podem coordenar os seus planos de ação e alcançar as suas metas.

As percepções, os valores, os sentimentos e as relações de poder que perpassam a prevenção da AIDS só são compreendidos quando se busca uma maior aproximação aos contextos reais do cotidiano, nos quais as mulheres se posicionam diante das propostas institucionais, definindo se são boas, funcionam e são do seu autêntico interesse. ${ }^{8}$ Existe sempre a possibilidade de conflitos entre os valores e interesses que orientam as decisões sobre a vida sexual e a saúde e aqueles que norteiam as recomendações 
programáticas, as quais colocam um dos mais críticos problemas para as mulheres: a negociação do sexo seguro. Necessidade que se conforma no interior de uma cultura que responsabiliza a mulher pela prevenção da gravidez, fortalece as opções contraceptivas que não protegem contra as doenças sexualmente transmissíveis e estimula a utilização de métodos que diminuem inclusive a importância do diálogo com o parceiro. ${ }^{9}$

Não se pode perder de vista que as diferenças de gênero aparecem nas formas de julgar e decidir sobre o que é e o que não é seguro e, de fato, adotar práticas protetoras. Como discutir a negociação do sexo seguro e as mudanças nos repertórios sexuais, sem levar em conta a representação da mulher no imaginário social e a assimetria de poder nas relações entre os sexos? Apesar das transformações significativas na atitude diante da sexualidade, em particular entre as jovens de classe média e alta que residem em grandes cidades, os lugares desiguais de homens e mulheres não sofreram alterações profundas. Isto não significa que as mulheres sejam destituídas de poder e que não disponham de estratégias e táticas para alcançar os fins pretendidos, mas, evidencia que são maiores os obstáculos com que se defrontam no processo de negociação e decisão sobre o uso do preservativo, principalmente quando mantêm um relacionamento duradouro. 9,10

A ação (ou omissão) frente à prevenção da AIDS dá a medida do espaço social das mulheres para, a partir da consciência sobre a suscetibilidade à doença, mudar as suas práticas. Desse modo, para enfrentar o desafio da educação em saúde, três aspectos adquirem importância fundamental: a) as pessoas têm possibilidades distintas de transformar a sua realidade frente à epidemia; b) elas devem ter ampla participação na escolha de meios e de fins quando se trata de se proteger do HIV; e c) quando se fala em participação é preciso assumir que o ponto a que se deseja chegar nunca estará decidido de uma vez por todas. ${ }^{11}$ Portanto, cabe a organização dos cuidados de saúde não subestimar a necessidade de produzir relações de entendimento sobre a melhor forma de atuar, recolocando criticamente as recomendações técnicas no contexto prático da vida das pessoas.

Embora pouco desenvolvidas, as reflexões sobre uma avaliação coerente com as necessidades comunicacionais das ações programáticas originaram a idéia de efetividade comunicacional, dizendo respeito à criatividade dos meios de trabalho para favorecer uma relação dialógica entre os sujeitos envolvidos. Para Sala et al. (1996: 191):12 "A principal motivação para tais explorações tem sido a crítica à definição estritamente instrumental na apreensão dos objetos para o trabalho em saúde e ao movimento de dessubjetivação decorrente desse instrumentalismo". Essa idéia, ainda muito genérica, amplia as possibilidades da avaliação estabelecer um diálogo dinâmico com o processo de trabalho, à medida que resgata aspectos não-materiais que, também, estão comprometidos com a realização de finalidades previamente estabelecidas para as práticas de saúde.

Neste estudo, tratando a dimensão relacional como elemento essencial das ações em saúde, pretende-se elucidar a fecundidade da prática educativa focalizada na prevenção da transmissão materno-fetal da AIDS para promover a comunicação entre as mulheres e os serviços. A inclusão de vários serviços, representando tipos distintos de demanda, modelos diferentes de organização e especificidades na execução do trabalho, ao permitir a apreensão de variações no processo de educação preventiva, potencializa a capacidade de evidenciar de modo mais seguro os aspectos essenciais do problema avaliado, aumentando a utilidade de seus resultados. É fundamental considerar esta aproximação como preliminar a um momento do processo de trabalho, a sua dimensão comunicativa, cuja complexidade requer explorações mais detalhadas.

\section{Métodos}

O trabalho aqui descrito é um estudo exploratório, parte de uma pesquisa mais ampla sobre a atenção pré-natal realizada, em 27 serviços da rede básica da Secretaria Municipal de Saúde da Cidade do Recife, PE, Brasil: 19 centros de saúde, duas maternidades e seis unidades de saúde da família (USF), com o propósito de avaliar a operacionalidade das ações de acompanhamento das mulheres grávidas, com ênfase na prevenção da transmissão materno-fetal do HIV. Recorreu-se à triangulação para ampliar as possibilidades de apreensão da realidade, por meio da articulação das abordagens quantitativa e qualitativa, da cooperação de usuárias, profissionais de saúde e gerentes, além da integração de diferentes técnicas de coleta de informações. 13

Neste estudo, o olhar se detém, ainda de modo preliminar, sobre a comunicação entre as usuárias e os serviços de saúde, no interior do processo de educação preventiva desenvolvida na atenção pré-natal. Esta pesquisa foi requerida pelo Programa de Doenças Sexualmente Transmissíveis/AIDS da Secretaria Municipal de Saúde do Recife. Os gerentes dos distritos sanitários e a direção de cada unidade selecionada autorizaram a sua realização. Para aplicação dos 
questionários individuais foi solicitado o consentimento verbal, informado e assegurado o caráter espontâneo e confidencial da participação das gestantes e dos profissionais - dois médicos se recusaram alegando falta de tempo.

Em cada um dos seis Distritos Sanitários da cidade do Recife: a) o número de centros de saúde selecionados é proporcional ao número de serviços dessa categoria que realiza o pré-natal, sendo alocados aqueles que tiveram, no ano de 1997, um maior número de consultas na atenção à gestante; b) escolheu-se a USF que atende a um maior número de famílias; e c) incluíram-se as maternidades com assistência pré-natal. O trabalho de campo foi feito, no período de maio a julho de 1998, por uma equipe constituída por médica sanitarista, enfermeira e assistente social, todas com experiência em avaliação.

Para a coleta de informações foram utilizados cinco instrumentos. O primeiro para descrever o modo de organização por meio de entrevistas com os gerentes e as equipes técnicas, esclarecendo a composição da equipe, as rotinas de trabalho e as programações. O segundo, para examinar o processo de trabalho na atenção à gestante, incluído um pequeno roteiro para observação direta sistemática das práticas educativas, focalizando o local em que realizavam as atividades coletivas, os conteúdos e as características da comunicação. Os pesquisadores de campo observaram o conjunto das ações durante no mínimo dois turnos, em dias da semana selecionados ao acaso, perfazendo um total de 214 horas. O terceiro, para delinear o perfil sócio-demográfico das 355 mulheres que aguardavam consulta pré-natal: idade, inserção no mercado de trabalho, experiência reprodutiva e acesso a algum tipo de orientação durante o acompanhamento. O quarto, para conhecer as percepções e práticas das mulheres acerca da prevenção da AIDS e as opiniões sobre a assistência pré-natal. Entrevistaram-se 81 gestantes, selecionadas entre as menores de 20 anos de idade (independente da paridade), de primeira gestação e com maior número de gestação. O quinto, para tomar conhecimento dos pontos de vista sobre a atuação do pré-natal, tendo participado 23 médicos, 13 enfermeiras, quatro psicólogas e duas assistentes sociais que estavam realizando atendimento durante as observações Haviam sido identificados 57 médicos, 31 enfermeiras, 12 psicólogas e seis assistentes sociais que realizavam atividades de pré-natal.

Para mapear os "núcleos de sentido" emergentes, as respostas às perguntas abertas foram organizadas e estudadas, tendo sido feita a opção por ressaltar qualitativamente a presença de determinados temas que expressam as concepções e os valores de referência dos discursos, portanto, não foi feita a contagem da freqüência das unidades de significação. ${ }^{14}$ A análise destacou: a) a racionalidade que predomina no processo de comunicação em saúde; b) as situações na produção dos serviços que podem ser tomadas como instrumentos das interações com as usuárias; e c) a referência espontânea, feita pelas mulheres e pelos profissionais, a elementos da tecnologia não-material do trabalho em saúde, tais como, as relações interpessoais e a criação de vínculos. Recorreu-se ao trabalho de dois avaliadores para ampliar a visão e afinar as possíveis divergências de interpretação. Na comparação da mediana de idade das gestantes atendidas nos três tipos de unidades, estabeleceu-se a existência de diferenças estatisticamente significativas por meio da análise de variância de Kruskal-Wallis.

\section{Resultados e Discussão}

Embora a dimensão educativa se constitua em uma parte essencial das práticas de saúde, em especial na unidade básica - porta de entrada para o sistema de assistência e um nível próprio de atendimento comprometido em atingir a maior integralidade possível por meio do trabalho de cada profissional e de uma equipe multiprofissional - salienta-se, nos serviços de atenção tradicional, em particular nos centros de saúde, a pouca valorização dessas atividades na atenção pré-natal. Em geral, o processo educativo é restrito ao fornecimento pelo médico, durante a consulta, de informações sobre alguns aspectos relacionados com a gravidez, o parto e os cuidados com o bebê. Como a consulta médica é o único momento interativo direto das mulheres com os profissionais, a dimensão educacional fica diluída no conjunto de práticas voltadas para a detecção e o controle de fatores de risco biológicos, tais como, a anamnese dirigida, o exame físico e a solicitação de exames para seguimento da gestação.

É inegável a maior sistematicidade das práticas educativas nas equipes de saúde da família, já que esta dimensão é parte da assistência individual e coletiva prestada pela unidade e do acompanhamento domiciliar realizado pelo agente comunitário de saúde. Porém, as atividades de grupos não ocorrem em todos os horários da atenção pré-natal em nenhum dos serviços onde informam efetuá-las $(48,1 \%)$ - todas USF, maternidades e $26,3 \%$ dos centros de saúde (em $40 \%$, exclusivamente para gestantes adolescentes). As repercussões sobre a captação das gestantes, no entanto, diferem de acordo com o modo de organização do processo de trabalho. Nas USF, as 
mulheres são orientadas para voltar à unidade nos dias previstos para o trabalho coletivo, enquanto na atenção tradicional, os grupos são formados com aquelas presentes na sala de espera.

Houve prática educativa coletiva, nas salas de espera, em seis dos 13 serviços que referem a sua realização (duas USF). A busca de favorecer a compreensão do conteúdo foi, na maioria desses serviços, uma preocupação dos profissionais responsáveis pela realização das palestras — na atenção tradicional, em geral, as enfermeiras e, no Programa de Saúde da Família (PSF), as enfermeiras e/ou os médicos - mas, simultaneamente, não foi feita a tentativa de introduzir no trabalho uma dinâmica de "conversa", incorporando a participação das mulheres. Essas não foram encorajadas para a troca de experiências e opiniões, falaram pouco, quase sempre fazendo perguntas com o intuito de esclarecer as dúvidas suscitadas pela exposição. Predominou a difusão unilateral de informações, sobressaindo à intenção de determinar o sentido que a proteção contra as doenças sexualmente transmissíveis e as vivências relacionadas com a maternidade devem assumir na vida do outro.

A inteligibilidade é um dos pressupostos básicos da comunicação, sem a qual não existe qualquer possibilidade de compreensão acerca do que foi dito, isto, independentemente de que os processos de comunicação se desenvolvam no plano da interlocução ou da transmissão de informações. ${ }^{7}$ Logo, no âmbito da educação em saúde, um dos maiores desafios que se colocam é a superação das barreiras impostas pelo jargão técnico, aspecto essencial para que haja compartilhamento de perspectivas normativas entre os profissionais de saúde e a população. Porém, o modo como se dá a prática educativa nos serviços estudados, torna-a mais próxima da visão estratégica e simplificadora que utiliza a linguagem do senso comum para tornar as mensagens mais atraentes, 4,5 minimizando a importância das formas diferenciadas de apropriação dos conhecimentos no dia a dia das pessoas.

Acresce-se ao caráter prescritivo da prática educativa, a escassez de informação sobre as doenças sexualmente transmissíveis e AIDS. Apenas 19,8\% das gestantes que participaram deste estudo - $45,4 \%$ nas USF, $28,3 \%$ nas maternidades e $12,1 \%$ nos centros de saúde, receberam algum tipo de orientação sobre a prevenção dessas enfermidades. A distribuição etária das mulheres mostra, segundo a Tabela 1, uma forte participação das menores de 20 anos $(33,0 \%)$, além da elevada concentração no grupo com idade entre 20-34 anos (64,0\%). A amplitude da distribuição inclui gestantes de 13 a 41 anos - não existindo diferença significativa entre as medianas de idade das usuárias dos três tipos de unidade $(\mathrm{H}=1,172 ; \mathrm{p}=0,557)$. Cerca de $18,0 \%$ trabalham, das quais $62,5 \%$ faltaram ao trabalho para se consultar. Para $45,1 \%$ essa é a primeira gestação e para $14,4 \%$, a quarta ou mais.

\begin{tabular}{|c|c|c|c|c|c|c|c|c|}
\hline \multirow{3}{*}{ Características } & \multicolumn{8}{|c|}{ Tipo de unidade } \\
\hline & \multicolumn{2}{|c|}{$\begin{array}{c}C S \\
(N=239)\end{array}$} & \multicolumn{2}{|c|}{$\begin{array}{c}\text { MAT } \\
(\mathrm{N}=48)\end{array}$} & \multicolumn{2}{|c|}{$\begin{array}{c}\text { PSF } \\
(N=68)\end{array}$} & \multicolumn{2}{|c|}{$\begin{array}{c}\text { Total } \\
(\mathrm{N}=355)\end{array}$} \\
\hline & $\mathrm{n}$ & $\%$ & $\mathrm{n}$ & $\%$ & $\mathrm{n}$ & $\%$ & $\mathrm{n}$ & $\%$ \\
\hline \multicolumn{9}{|l|}{ Idade (anos) } \\
\hline $13-19$ & 81 & 33,9 & 14 & 29,2 & 24 & 35,3 & 119 & 33,5 \\
\hline $20-34$ & 149 & 62,3 & 34 & 70,8 & 43 & 63,2 & 226 & 63,7 \\
\hline $35-41$ & 9 & 3,8 & - & - & 1 & 1,5 & 10 & 2,8 \\
\hline \multicolumn{9}{|c|}{ Inserção no mercado de trabalho } \\
\hline $\operatorname{Sim}$ & 50 & 20,9 & 5 & 10,4 & 9 & 13,2 & 64 & 18,0 \\
\hline Não & 189 & 79,1 & 43 & 89,5 & 59 & 86,9 & 291 & 82,0 \\
\hline \multicolumn{9}{|c|}{ Número de gestações } \\
\hline Uma & 108 & 45,2 & 22 & 45,9 & 30 & 44,1 & 160 & 45,1 \\
\hline Duas & 63 & 26,3 & 16 & 33,3 & 14 & 20,6 & 93 & 26,1 \\
\hline Três & 32 & 13,4 & 4 & 8,3 & 15 & 22,1 & 51 & 14,4 \\
\hline Quatro e + & 36 & 15,1 & 6 & 12,5 & 9 & 13,2 & 51 & 14,4 \\
\hline
\end{tabular}

CS = Centro de Saúde; MAT = Maternidade; PSF = Programa de Saúde da Família. 
Nenhuma dessas unidades faz o aconselhamento, mesmo aquelas que dizem informar sobre o exame anti-HIV (48,1\%, todas da atenção tradicional). As equipes de saúde, à parte o tipo organizacional, não estão capacitadas para desenvolver este trabalho de escuta ativa, individualizado, centrado no paciente. O despreparo dos serviços para ter o impacto esperado sobre a transmissão materno-fetal da AIDS convive com a falta de percepção das mulheres sobre a vulnerabilidade pessoal, pois, $98,0 \%$ mencionam o uso do preservativo masculino como medida para evitar a transmissão do vírus, mas, apenas $11,1 \%$ utilizam-no de modo sistemático. A não utilização do preservativo masculino é atribuída, fundamentalmente, a confiança na fidelidade do parceiro, a qual em algumas situações se acrescem outros motivos, tais como, os incômodos provocados pela camisinha e a recusa do parceiro.

"Meu marido trabalha viajando, aí logo que ele chega gosto de usar camisinha, mas no passar do tempo, a gente dá uma folguinha. Vivo só eu e meu marido, ele não tem relações fora".

Por enquanto, não, que eu sou casada. Eu sei que a gente corre muito perigo mesmo sendo casada, mas, até agora não faço nada para me proteger. Eu acho que até agora não é preciso, porque até agora ele nunca me deu motivo de ver e nem tão pouco de desconfiar".

"Meu esposo não usa camisinha, a gente usou uns dois meses, mas eu tinha relações e ficava toda ardida e também eu não acho que ele, ele é um homem crente a gente sempre confia mais".

"Porque o homem que eu vivo com ele não gosta de usar. Ele é uma pessoa de idade, não vive saindo com nenhuma mulher, aí ele não usa não".

As mulheres que dizem utilizar o preservativo em todas as relações sexuais, também, mostram às dificuldades para garantir o seu uso regular.

"Às vezes ele não quer, ele diz que eu tenho que confiar nele, aí eu, que estou a nove anos com ele, tenho que facilitar. No começo ele achou ruim, ele não quis usar, dizendo que não sentia prazer com a camisinha, ele botou mil dificuldades, mas, agora já se acostumou e pronto".

É motivo de grande preocupação o grande silêncio dos profissionais sobre as necessidades de comunicação que atravessam as práticas de saúde, em um contexto em que a prevenção da AIDS solicita que se reitere a compreensão das interações entre usuárias e serviços como um componente essencial da qualidade e dos resultados que podem ser atribuídos às ações de saúde. 15 Isso, certamente, tem repercussões negativas sobre a efetividade comunicacional, 12 ou seja, sobre a utilização dos saberes que operam no processo de trabalho em saúde como mediadores do "encontro" entre serviços e usuários, considerando-o como um momento interativo direto em que estes sujeitos trocam experiências e conhecimentos.

As limitações existentes na operacionalização do processo educativo na assistência pré-natal, praticamente, não provocam comentários das equipes: na atenção tradicional, apenas as psicólogas, e no PSF, sobretudo as enfermeiras, se mostram preocupadas com a não aderência das gestantes às orientações dadas e atribuem essa atitude à "falta de consciência" daquelas que estão sendo acompanhadas. Ao mesmo tempo em que, destacando as diferenças na percepção acerca das necessidades, entre as mulheres a importância conferida à educação em saúde se manifesta, nos centros de saúde, como crítica pela falta de informação e, nas unidades de saúde da família, como satisfação pela realização de palestras.

"Gosto das palestras, a gente se distrai $e$ aprende um pouco, das consultas que é um pouco demorada, mas, é porque examina bem, faz como é para fazer. Das agentes de saúde que são muito atenciosas, sem exceção de nenhuma".

"A doutora é boazinha, gosto da conversa que ela fala, da palestra que todo mês ela faz com a gente".

As relações interpessoais são bastante valorizadas pelas mulheres que, independentemente do tipo organizacional, externam a satisfação com o modo como são atendidas e com as características do exame físico.

"As pessoas aqui atendem muito bem, nunca fui maltratada".

"A atenção que a médica dá quando escuta a barriga, mede, faz perguntas, vê se o nenê está mexendo".

"Toda vez que a gente chega aqui é pesada, tira a pressão, tem até nutricionista. Gosto do atendimento do médico, ele é muito atencioso com a gente".

O processo educativo na prevenção da AIDS é, também, uma ação interpessoal que põe em jogo os antagonismos de preferência e os conflitos de valores e pressupõe a definição de regras para a convivência entre as mulheres e os agentes do trabalho em saúde. A criação de vínculos entre cada usuário e uma equipe e/ou um profissional, mais do que a simples adscrição a um serviço ou a inscrição formal a um programa, é uma relação contínua, pessoal, de confiança. 16 Essa relação é fundamental para a reflexão conjunta sobre estratégias preventivas viáveis, processo que passa, necessariamente, pela possibilidade das mulheres e dos profissionais se colocarem 
face a face, envolvendo-se nas negociações sobre o sentido e a validez de discursos e práticas.

\section{Conclusões}

Os resultados sugerem que existe pouco interesse prático dos profissionais de saúde em participar da construção compartilhada de estratégias por meio das quais as mulheres, seus parceiros e seus filhos possam estar protegidos do HIV. De um lado, o repasse de informações mostra que os serviços tendem a adotar um modelo de trabalho educativo que faz prevenção para o outro, ou seja, a educação em saúde continua se desenvolvendo como "monólogo tecnocrático", 15 subestimando o fato de que as mulheres devem ser colaboradoras e partícipes na escolha de meios e fins para o enfrentamento da infecção pelo HIV.11 De outro, a necessidade premente dos sujeitos envolvidos desenvolverem uma maior consciência de suas possibilidades, de seus limites e de suas autênticas aspirações, encontrando soluções criativas para vencer os desafios cotidianos da transformação dos hábitos e das condições de saúde, com repercussões positivas sobre a prevenção da AIDS.8,11,17

As deficiências da educação em saúde na prevenção materno-fetal da AIDS adquirem uma importância ainda maior quando se consideram as limitações da eleição da gravidez como o momento para detectar a infecção pelo HIV em mulheres — a doença é uma questão de todas as mulheres (e dos seus parceiros) que extrapola as fronteiras delimitadas pela gestação - e do modelo de organização da atenção que não tem sido capaz de responder às necessidades globais da saúde da mulher em especial quando se tem conhecimento de que as atividades de prevenção e tratamento das doenças sexualmente transmissíveis, cuja ocorrência aumenta as possibilidades de adquirir e transmitir o HIV, são as menos desenvolvidas no conjunto das práticas direcionadas à população feminina. 10 No cotidiano dos serviços, a dimensão educativa e comunicacional do cuidado com a saúde da mulher não têm merecido a valorização que a realidade da AIDS solicita.

É somente quando o trabalho educativo se empenha na construção de uma vida consciente e assumida criticamente, comprometida com a contemplação do outro e da diversidade, que se defronta com a sua grande questão, justamente, a de fazer avançar o diálogo entre as distintas lógicas argumentativas. Tal procedimento implica na reestruturação das relações entre os profissionais de saúde e as mulheres, pressupondo um processo de interpretação cooperativo por meio do qual se busca alcançar um acordo voluntário, isto é, a possibilidade de dar o espaço devido a cada uma das contribuições necessárias para enfrentar o problema da AIDS.8 Portanto, destaca a face mais humana, a dimensão intersubjetiva do cuidado de saúde, o que desloca a complexidade da atuação do elemento técnico para o relacional, requerendo o aumento do tempo da atenção, da conversa, da troca de experiências.

\section{Agradecimentos}

Às pesquisadoras de campo: Dionila Ferreira de Andrade, Maria das Dores Teixeira da Câmara e Suzette Maria Feitosa Brito.

\section{Referências}

1. Veloso VG, Vasconcelos AL, Grinsztein B. Prevenção da transmissão vertical no Brasil. Bol Epidemiol 1999; 12: 16-25.

2. Paiva V. Revendo as primeiras abordagens para a prevenção da Aids. In: Paiva V. Fazendo arte com a camisinha. São Paulo: Summus; 2000. p. 27-53.

3. Parker R. Mudanças de paradigmas na intervenção e pesquisa de HIV/AIDS: comportamento, cultura e política. In: Parker R. Na contramão da AIDS: sexualidade, 
intervenção, política. Rio de Janeiro: ABIA; 2000. p. 65 78

4. Pitta AMR. Interrogando os campos da saúde e da comunicação: notas para o debate. In: Pitta AMR, organizadora. Saúde \& comunicação: visibilidades e silêncios. São Paulo: Hucitec; 1995. p. 239-66.

5. Teixeira RR. Modelos comunicacionais e práticas de saúde. Interface: Comun Saúde Educ 1997; 1: 7-40.

6. Schraiber LB. No encontro da técnica com a ética: o exercício de julgar e decidir no cotidiano do trabalho em Medicina. Interface: Comun Saúde Educ 1997; 1: 123-38.

7. Habermas J. Teoría de la acción comunicativa. 2. ed. Madrid: Taurus; 1988

8. Feliciano KVO. Prevenção da AIDS entre jovens: conflitos entre os discursos técnicos e a prática cotidiana de escolares da Região Metropolitana do Recife [tese doutorado]. São Paulo: Departamento de Medicina Preventiva, Faculdade de Medicina da Universidade de São Paulo; 2001.

9. Vilella WV. Prevenção do HIV/AIDS, gênero e sexualidade: um desafio para os serviços de saúde. In: Barbosa RM, Parker R, organizadores. Sexualidades pelo avesso: direitos, identidades e poder. Rio de Janeiro: Instituto de Medicina Social da Universidade Estadual do Rio de Janeiro; 1999. p. 199-213.

10. Barbosa R. Lago TG. AIDS e direitos reprodutivos: para além da transmissão vertical. In: Parker R. organizador. Políticas, instituições e AIDS: enfrentando a epidemia no Brasil. Rio de Janeiro: Zahar; 1997. p.63-75.
11. Ayres JRCM. Cidadania, vulnerabilidade e prevenção de HIV/AIDS. In: Pinto T, Telles IS, organizadores. AIDS e escola: reflexões e propostas do EDUCAIDS. São Paulo: Cortez; 2000. p. 21-8.

12. Sala A, Nemes MIB, Cohen DD. Avaliação na prática programática. In: Schraiber LB, Mendes-Gonçalves RB, Nemes MIB, organizadores. Saúde do adulto, programas e ações na unidade básica. São Paulo: Hucitec; 1996. p. 173-93.

13. Minayo MCS. O desafio do conhecimento: pesquisa qualitativa em saúde. 2. ed. São Paulo: Hucitec; 1993.

14. Bardin L. Análise de conteúdo. Lisboa: Edições 70; 1977.

15. Rodrigues LAJ, Kayano R, Lippe TL, Schraiber LB. Interações com usuários na unidade básica de saúde. In: Schraiber LB, Mendes-Gonçalves RB, Nemes MIB, organizadores. Saúde do adulto, programas e ações na unidade básica. São Paulo: Hucitec; 1996. p. 262-75.

16. Cecílio LCO. As necessidades de saúde como conceito estruturante na luta pela integralidade e eqüidade na atenção em saúde. In: Pinheiro R, Mattos RA. organizadores. Os sentidos da integralidade na atenção e no cuidado de saúde. Rio de Janeiro: Instituto de Medicina Social da Universidade Estadual do Rio de Janeiro; 2001. p. 113-26.

17. Merchán-Hamann E. Os ensinos da educação para a saúde na prevenção de HIV-Aids: subsídios teóricos para a construção de uma práxis integral. Cad Saúde Pública 1999; 15 (Supl 2): 85-92.

Recebido em 4 de junho de 2003

Versão final reapresentada em 18 de agosto de 2003

Aprovado em 19 de novembro de 2003 